\title{
Elaboração de massa alimentícia fresca sem glúten enriquecida com farinha de resíduo de açaí (Euterpe oleracea Mart.) e bacaba (Oenocarpus bacaba Mart.)
}

Preparation of fresh gluten free pasta enriched with açaí waste flour (Euterpe oleracea Mart.) and bacaba (Oenocarpus bacaba mart.)

Preparación de pasta fresca sin gluten enriquecida con harina de residuo de açaí (Euterpe oleracea Mart.) y bacaba (Oenocarpus bacaba Mart.)

Samara Kelly Amaral Barros ORCID: https://orcid.org/0000-0002-7090-8650 Universidade Federal do Tocantins, Brasil E-mail: aramasly@gmail.com

Adriana Régia Marques de Souza ORCID: https://orcid.org/0000-0002-0608-9988 Universidade Federal de Goiás, Brasil E-mail: adriana_regia_souza@ufg.br Flávio Santos Silva

ORCID: https://orcid.org/0000-0003-3058-0969 Universidade Federal do Tocantins, Brasil E-mail: engalflavio@yahoo.com.br

Caroline Roberta Freitas Pires ORCID: https://orcid.org/0000-0002-1427-7276 Universidade Federal do Tocantins, Brasil E-mail: carolinerfpires@uft.edu.br

Clarissa Damiani

ORCID: https://orcid.org/0000-0001-8507-0320 Universidade Federal de Goiás, Brasil E-mail: clarissadamiani@uft.br

Miriam Fontes Araújo Silveira ORCID: https://orcid.org/0000-0002-0191-8628 Universidade Federal de Goiás, Brasil E-mail: miriamfas.ufg@gmail.com

Camila Rodrigues Evangelista Silva ORCID: https://orcid.org/0000-0001-9463-030X Universidade Federal do Tocantins, Brasil

E-mail: camillarodrigues232011@gmail.com

\section{Resumo}

O presente estudo teve por objetivo desenvolver e caracterizar massas alimentícias fresco tipo talharim, com substituição parcial da farinha de arroz pela farinha do caroço de açaí FCA e da casca da bacaba FCB, e avaliar suas características físico-químicas, composição proximal, cor, propriedades tecnológicas e perfil de textura. Para isso, foram elaboradas cinco formulações de massas, em substituição massa controle (MC), contendo 5\% (FCA), 10\% (FCA), 5\% (FCB) e 10\% (FCB). A umidade das massas alimentícias apresentou um percentual máximo de 35\%, assim as mesmas estão de acordo com a legislação brasileira vigente para massa fresca. As formulações das massas alimentícias com FCA apresentaram teores maiores para proteínas e cinza na $10 \%$. Para as formulações de FCB mostrou maior teor em lipídeos. Em relação aos valores energéticos as massas alimentícias enriquecidas com a FCA e FCB podem ser vistas como um alimento rico em calorias, de acordo com a legislação brasileira. Quanto ás propriedades tecnológicas, as adições das farinhas nas massas causaram diferença estatística no parâmetro do aumento da massa. A análise de cor, os parâmetros chroma $\left(\mathrm{C}^{*}\right)$ e o ângulo hue $\left(\mathrm{H}^{*}\right)$ obtiveram resultados que interferiram na cor indicando à tonalidade vermelho puro para ambas massas alimentícias FCA e FCB. Com relação aos parâmetros de textura, dureza, gomosidade e mastigabilidade todas as concentrações de FCA e FCB apresentaram diferença significativa comparando-a com a MC. As massas alimentícias elaboradas com diferentes concentrações da FCA e FCB proporcionaram variações de ingredientes, além de contribuir com o meio ambiente, visto que o descarte de resíduo será reduzido.

Palavras-chave: Massa fresca; Resíduo de caroço; Resíduo de casca; Desenvolvimento de novos produtos. 


\begin{abstract}
The present study aimed to develop and characterize fresh pasta type noodles, with partial replacement of rice flour by the flour of the açaí seed FCA and of the FCB bacaba shell, and to evaluate its physical-chemical characteristics, proximal composition, color, technological properties and texture profile. For this purpose, five mass formulations were prepared, replacing control mass (MC), containing 5\% (FCA), 10\% (FCA), 5\% (FCB) and 10\% (FCB). The humidity of the pasta presented a maximum percentage of $35 \%$, so they are in accordance with the Brazilian legislation in force for fresh pasta. Pasta formulations with FCA showed higher levels for protein and ash at 10\%. For FCB formulations it showed a higher content of lipids. Regarding energy values, pasta enriched with FCA and FCB can be seen as a food rich in calories, according to Brazilian legislation. As for technological properties, the addition of flours to the doughs caused a statistical difference in the parameter of the increase in dough. The color analysis, the chroma parameters $(\mathrm{C} *)$ and the hue angle $\left(\mathrm{H}^{*}\right)$ obtained results that interfered with the color, indicating the pure red tint for both FCA and FCB pasta. Regarding the parameters of texture, hardness, guminess and chewability, all concentrations of FCA and FCB showed a significant difference when compared to MC. Pasta made with different concentrations of FCA and FCB has provided variations in ingredients, in addition to contributing to the environment, since waste disposal will be reduced.
\end{abstract}

Keywords: Fresh pasta; Core residue; Bark residue; Development of new products.

\title{
Resumen
}

El presente estudio tuvo como objetivo desarrollar y caracterizar fideos tipo pasta fresca, con sustitución parcial de harina de arroz por la harina de semilla de açaí FCA y de cáscara de bacaba FCB, y evaluar sus características físicoquímicas, composición proximal, color, propiedades tecnológicas características y perfil de textura. Para ello, se prepararon cinco formulaciones de masa, reemplazando la masa de control (MC), conteniendo 5\% (FCA), 10\% (FCA), $5 \%$ (FCB) y $10 \%$ (FCB). La humedad de la pasta presentó un porcentaje máximo del 35\%, por lo que están de acuerdo con la legislación brasileña vigente para la pasta fresca. Las formulaciones de pasta con FCA mostraron niveles más altos de proteína y cenizas al 10\%. Para las formulaciones de FCB, mostró un mayor contenido de lípidos. En cuanto a los valores energéticos, la pasta enriquecida con FCA y FCB puede verse como un alimento rico en calorías, según la legislación brasileña. En cuanto a las propiedades tecnológicas, la adición de harinas a las masas provocó una diferencia estadística en el parámetro del aumento de masa. El análisis de color, los parámetros de croma $(\mathrm{C} *)$ y el ángulo de tono $(\mathrm{H} *)$ obtuvieron resultados que interfirieron con el color, indicando el tono rojo puro tanto para la pasta FCA como para la FCB. En lo que respecta a los parámetros de textura, dureza, gomosidad y masticabilidad, todas las concentraciones de FCA y FCB mostraron una diferencia significativa en comparación con el MC. La pasta elaborada con diferentes concentraciones de FCA y FCB proporcionó variaciones en los ingredientes, además de contribuir al medio ambiente, ya que se reducirá la eliminación de desechos.

Palabras clave: Pasta fresca; Residuo de núcleo; Residuos de cáscara; Desarrollo de nuevos produtos.

\section{Introdução}

A massa alimentícia é o produto não fermentado, obtido pelo amassamento da farinha de trigo e/ou produtos de trigo duro e/ou outros cereais, leguminosas, raízes ou tubérculos, (Brasil, 2005). As massas frescas apresentam características artesanais, denotando umidade máxima de 35\%, uma vez que passam por um processo parcial de secagem, e sendo comercializadas sob refrigeração, mantendo suas características de textura e sabor semelhantes aos das massas caseiras (Brasil, 2000; ABIMAPI, 2019).

O Brasil é o terceiro maior produtor mundial de massas alimentícias. Esse significativo está relacionado a versatilidade do produto, por um custo relativamente baixo, além de ser prático, rápido e atrativo (ABIMAPI, 2019). No ano de 2018 o consumo per capto médio foi de 4,39 kg correspondendo uma produção de 0,892 milhões de toneladas de massas alimentícias.

É crescente o interesse no desenvolvimento de alimentos isentos de glúten, uma vez que algumas pessoas são sensíveis ao mesmo. A doença celíaca apresenta danos de base imunológica, além de estimular outras doenças, como osteoporose, que é considerada uma preocupação para as agências de saúde pública (Sanguinetti et al, 2016; Soler et al, 2016). Sob esse cenário, houve um aumento na busca por alimentos livre de glúten que também é associada a um estilo de vida mais saudável. As farinhas de cereais sem glúten, podem ser utilizadas no processamento de alimentos como substituto para a farinha de trigo. Asmeda et al. (2016) e Phongthai et al. (2017) afirmam que a adição de farinha de arroz para elaboração de produtos é interessante por possuir baixos níveis de sódio melhora a digestibilidade. Diante disso, os resíduos 
descartados do processamento de vegetais podem ser aproveitados na elaboração de novas formulações alimentícias isentas de glúten, visando diminuição de custos das formulações, desperdícios de alimentos, na redução dos impactos, além de contribuir para o combate à desnutrição e à fome (Silva, et al, 2019).

Dentre as frutas que apresentam perdas durante o processamento estão o açaí (Euterpe oleracea Mart) e a bacaba (Oenocarpus bacaba Mart.), ambos são frutos roxos escuros, pertencente à família botânica Arecaceae. É uma palmeira nativa dos biomas Amazônica e Cerrado, com alto potencial de exploração (Abadio Finco et al, 2012). A parte comestível do açaí, direcionada a produção de suco e polpa congelada, representa apenas $10 \%$ do fruto. O endocarpo do fruto é volumoso, duro, envolvido por um tecido fibroso e envolto por uma camada de polpa fina e seca, denotando um elevado valor de fibras (De Freitas Bonomo et al, 2014). Para a bacabeira o seu fruto é consumido de forma de suco, polpa e muito apreciado em nível regional, possui um sabor agradável e uma cor creme leitosa (Nascimento et al, 2019). Pesquisas indicam que a fruta apresenta alto teor de lipídios, uma concentração elevada de fibra e sua polpa um produto com pouco ácido (Seixas et al, 2016; Neves et al, 2015).

Nesse sentido, a utilização do resíduo do açaí e da bacaba para fabricação de farinha e o desenvolvimento de uma massa alimentícia, possibilitaria a aceitação deste produto para atender ao público celíaco e com a exigência de produtos sem glúten, também contribuiria para a diminuição do descarte do resíduo. Diante do exposto o presente trabalho teve como objetivo o desenvolvimento e caracterização de massas alimentícias fresca, tipo talharim, com substituição parcial da farinha de arroz pela a farinha do caroço de açaí e da casca da bacaba, e avaliar suas características físico-químicas, composição proximal, cor, propriedades tecnológicas e perfil de textura.

\section{Material e Métodos}

\subsection{Obtenção das farinhas}

Os caroços de açaí foram adquiridos na cidade de Imperatriz (MA). Já os frutos da bacaba foram adquiridos na Aldeia Pedra Branca Terra Indígena Krahô Itacajá - TO. No momento da colheita, as bacabas foram retiradas por cacho, e em seguida, armazenadas em saco plástico. A preparação do experimento ocorreu dentro de um período de 24 horas após a retirada dos frutos. Ambas matérias-primas, foram transportadas para Laboratório de Tecnologia de Alimentos da Universidade Federal do Tocantins, Campus Palmas (TO), onde foram submetidos à lavagem com água corrente e em seguida colocadas numa solução de hipoclorito de sódio ( $\mathrm{NaClO}$ ) a 200 ppm por 15 minutos e, após foram lavados em água corrente para retirar o excesso de cloro, de acordo com a Resolução da Agência de Vigilância Sanitária - RDC nº 216/2004 (Brasil, 2004). Para obtenção das cascas do fruto da bacaba o despolpamento foi feito manualmente. Depois da limpeza, as frutas foram submersas em água ultra-pura a $80^{\circ} \mathrm{C}$ por 30 minutos para remoção manual das cascas, o material removido foi seco imediatamente em estufa.

Os caroços de açaí e as cascas das bacabas foram acomodados em bandejas e levados para estufa de circulação forçada de ar do modelo (SL -102) marca Ethik e secas a $50^{\circ} \mathrm{C}$, até $15 \%$ de umidade, conforme a RDC 263/2005 (Brasil, 2005).

Os caroços foram secos em 28 horas e as cascas das bacabas em 45 horas. Posteriormente, as amostras dos caroços secos foram trituradas, utilizando um moinho de facas, tipo Willye (modelo STAR FT- 50). Após serem triturados foram passados em moinho de martelo (Vieira MCS $280(05 \mathrm{cv})$ ) nas peneiras de furo de $0,7 \mathrm{~mm}$ e de $0,3 \mathrm{~mm}$. As cascas, após a secagem, foram trituradas usando um liquidificador (Mondial Turbo, L-1200 BI) e, em seguida foram moídas moinho de martelo com a peneira do furo $0.3 \mathrm{~mm}$. Os produtos dos resíduos retirados das peneiras foram classificados como farinhas.

A (FCA) foi acondicionada em sacos plásticos de média densidade (PEMD), de $1 \mathrm{~kg}$. Para a farinha da casca da bacaba (FCB) foi fracionada em porções de 100g e acondicionada em saco Stand Up Pouch Metalizado. As duas farinhas 
foram seladas a quente em seladora comercial, sendo da FCA armazenada à temperatura ambiente e FCB armazenado em dessecador.

\subsection{Processamento das massas alimentícias}

As massas alimentícias foram desenvolvidas no Laboratório de Frutas e Hortaliças na Universidade Federal do Tocantins no campus de Palmas (TO).

\subsubsection{Matérias primas utilizadas}

Para obtenção do produto final e da amostra controle, ensaios de formulações preliminares foram realizados para padronização dos ingredientes: goma xantana, albumina, farelo de linhaça, farinhas de resíduos do caroço de açaí e da casca da bacaba, farinha de arroz, água e especiarias. Todos os ingredientes, exceto as farinhas de resíduos, foram adquiridos em comércio local. Como estabilizante e emulsificante utilizou-se a goma xantana dentro da quantidade permitida pela RDC n. 45, de 03 de novembro de 2010 (Brasil, 2003), que é de 1,0 g/100g.

Condições ideais foram verificadas para o desenvolvimento das formulações da massa, de forma que a mesma pudesse ser aberta sem quebrar e que obtivesse o formato mais adequado ao objetivo proposto. Foram desenvolvidas 03 formulações do produto final para cada farinha de resíduo obtida (farinha da casca da bacaba e caroço do açaí): massa controle (MC) em que não há adição de resíduos; formulação com adição de 5\% (F1) e de 10\% (F2) de farinha de resíduo. Pode-se observar nas Tabelas 1 e 2 as formulações das massas alimentícias frescas da farinha do caroço do açaí (FCA) e farinha da casca da bacaba (FCB).

Tabela 1. Formulações das massas alimentícias frescas produzidas com farinha de caroço de açaí (FCA).

\begin{tabular}{cccc}
\hline Ingredientes & \multicolumn{3}{c}{ Formulações } \\
\cline { 2 - 4 } & MC & F1 & F2 \\
\cline { 2 - 4 } Farinha de arroz $(\mathrm{g})$ & 100 & 87,5 & 83,5 \\
FCA $(\mathrm{g})$ & - & 5 & 10 \\
Goma xantana $(\mathrm{g})$ & 1,0 & 1,0 & 1,0 \\
Albumina $(\mathrm{g})$ & 5,0 & 5,0 & 5,0 \\
Água $(\mathrm{ml})$ & $95 \mathrm{~mL}$ & $90 \mathrm{~mL}$ & $85 \mathrm{~mL}$ \\
Farelo de linhaça $(\mathrm{g})$ & 2,0 & 2,0 & 2,0 \\
\hline
\end{tabular}

*Massa controle - (MC); F1 (5\% de FCA); F2 (10\% de FCA). Fonte: Autores (2021).

Tabela 2. Formulações da massa alimentícias frescas produzidas com farinha de casca de bacaba (FCB).

\begin{tabular}{cccc}
\hline Ingredientes & & \multicolumn{2}{c}{ Quantidade (100g de massa) } \\
& MC & F1 & F2 \\
\cline { 2 - 4 } Farinha de arroz $(\mathrm{g})$ & 100 & 87,5 & 83,5 \\
FCB $(\mathrm{g})$ & - & 5 & 10 \\
Goma xantana $(\mathrm{g})$ & 1,0 & 1,0 & 1,0 \\
Albumina $(\mathrm{g})$ & 5,0 & 5,0 & 5,0 \\
Água $(\mathrm{ml})$ & $95 \mathrm{~mL}$ & $75 \mathrm{~mL}$ & $75 \mathrm{~mL}$ \\
Farelo de linhaça $(\mathrm{g})$ & 2,0 & 2,0 & 2,0 \\
\hline
\end{tabular}

*Massa controle - (MC); F1 (5\% de FCB); F2 (10\% de FCB). Fonte: Autores (2021).

Foi realizado o amassamento manual dos ingredientes ( 9 a $11 \mathrm{~min}$ ) para formar uma estrutura de massa homogênea. A massa foi moldada e deixada para descansar enrolada em filme de plástico PVC por um período de 5 horas na temperatura $21^{\circ} \mathrm{C}$. Após esse período, a massa foi aberta com um rolo manual e passada numa máquina de massa de operação manual 
(Florença) sendo posteriormente cilindrada em superfície untada com farinha de arroz até obter uma espessura de aproximadamente $5 \mathrm{~mm}$. Seguidamente, a massa foi cortada no formato de talharim com espessura de aproximadamente 0,5 com de largura e $15 \mathrm{~cm}$ de comprimento. A massa foi acondicionada em bandeja de isopor M-57, embalada em filme de plástico PVC e em seguida armazenada em geladeira por 24 horas à temperatura de $4^{\circ} \mathrm{C}$ até realização das análises.

\subsection{Caracterização das massas alimentícias frescas enriquecidas com FCA e FCB}

A caracterização físico-química foi realizada no Laboratório de Análises de Alimentos (LANA) e a determinação de cor no Laboratório de Frutas e Hortaliças da Universidade Federal de Tocantins - UFT. A análise de textura foi realizada no Laboratório Multiusuário, da Escola de Agronomia, da Universidade Federal de Goiás - UFG.

\subsection{1 pH, acidez total titulável e sólidos solúveis ( $\left.{ }^{\circ} \mathrm{Brix}\right)$}

Para a determinação do $\mathrm{pH}$ foram pesados aproximadamente $5 \mathrm{~g}$ da amostra base úmida, após foram adicionados 45 $\mathrm{mL}$ de água destilada em béquer de $100 \mathrm{~mL}$ procedendo a homogeneização, com auxílio de um bastão de vidro. Em seguida foi realizada a leitura do $\mathrm{pH}$ em potenciômetro digital da marca DigimedR modelo DM-22 calibrado com a solução tampão 4,0 e 7,0. A acidez titulável (TA, g ácido cítrico $100 \mathrm{~g}$-1) foi determinada por titulação, utilizando $0,1 \mathrm{~N}$ usando fenolftaleína a $1 \%$ como indicador. Os parâmetros foram determinados de acordo com os métodos da Associantion of Official Analytical Chemistry (A0AC, 20212).

Os sólidos solúveis totais foram determinados utilizando-se um refratômetro com compensação de temperatura automática a $25^{\circ} \mathrm{C}$ e expressos ${ }^{\circ}$ Brix em segundo (AOAC, 2012).

\subsubsection{Composição proximal}

A análise proximal fundamentou-se na determinação de umidade presente nas amostras das massas alimentícias, realizada em estufa de circulação de ar com $105^{\circ} \mathrm{C}$, já para realização das análises de proteína, cinzas, lipídios e fibra bruta a umidade das massas alimentícias foi seca na temperatura de $60^{\circ} \mathrm{C}$ de acordo com a AOAC (2005). As demais análises de lipídios, cinzas e proteínas foram realizadas conforme metodologia proposta pela AOAC (2012).

A quantificação da fibra bruta foi realizada pelo método gravimétrico com digestão ácida da amostra com ácido sulfúrico ( $\left.\mathrm{H}_{2} \mathrm{SO} 4\right)$, de acordo com a AOAC (2000). A determinação de fração glicídica feita por diferença, segundo Brasil (2003). O valor energético total (VET) foi estimado em kcal/100g com os fatores de conversão Atwater e Woods (1906), 4kcal/g para carboidratos, $4 \mathrm{kcal} / \mathrm{g}$ para proteínas e $9 \mathrm{kcal} / \mathrm{g}$ para lipídios.

\subsection{Análises tecnológicas das massas alimentícias}

\subsubsection{Tempo ótimo de cozimento (TOC)}

O TOC foi realizado de acordo com o American Association of Cereal Chemists AACC 666-50 (2000). A cocção de $10 \mathrm{~g}$ de massa foi cozida em $500 \mathrm{~mL}$ de água em ebulição e o tempo ótimo foi aferida pela compressão da amostra do produto cozido, a cada 2 minuto, entre duas lâminas de vidro até o desaparecimento do eixo central.

\subsubsection{Percentual de aumento de massa ou rendimento do cozimento (AM)}

O AM foi determinado de acordo com o método 16-50 da AACC (2000). É determinado pela relação entre 10 gramas de massa fresca e sua massa após a cocção, usando- se o tempo ótimo de cozimento de cada amostra. Os valores foram expressos em porcentagem. 


\subsubsection{Textura das Massas Alimentícias (TPA)}

As massas produzidas foram submetidas à análise de textura (cisalhamento, dureza, adesividade, elasticidade, coesividade, gomosidade, mastigabilidade e resiliência) utilizando- se um texturômetro (Texture Analyser, TA-XT Plus, Surrey, England).

As massas cozidas foram cortadas com $3 \mathrm{~cm}$ de comprimento e realizadas as leituras referentes à força de cisalhamento para cortar a massa, indicando a firmeza das diferentes massas alimentícias. As condições de operação do texturômetro para a medida da força em compressão foram: velocidade de pré-teste de $0,5 \mathrm{~mm} \mathrm{~s}-1$, velocidade de teste de 0,17 mm s- 1, velocidade de pós-teste de 10,0 mm s-1, distância de 0,3 mm s-1, trigger: base (altura inicial de $5 \mathrm{~mm}$ ) e Probe: HPP/BSG - Bland Set With Guillotine. A análise foi realizada a uma temperatura de $25^{\circ} \mathrm{C}$ (Andrade et al, 2015).

Para dupla compressão das amostras utilizou-se uma probe de alumínio (P/5) os seguintes parâmetros foram ajustados no equipamento: velocidade de teste: velocidade de pré-teste de $1,0 \mathrm{~mm} / \mathrm{s}$, velocidade de teste de $0,5 \mathrm{~mm} / \mathrm{s}$, velocidade de pósteste de $10 \mathrm{~m} / \mathrm{s}$, distância de 50\%, força: $3 \mathrm{~mm}$, tempo de compressão de $2 \mathrm{~s}$. As amostras foram comprimidas duas vezes para a obtenção dos parâmetros de textura (dureza, coesividade, elasticidade, gomosidade e mastigabilidade e resiliência). As análises foram realizadas em uma temperatura de $25^{\circ} \mathrm{C}$ (Andrade et al, 2015).

\subsection{Análises estatísticas}

Os dados coletados inicialmente foram inseridos em planilha desenvolvidas com o programa Microsolf Excel, versão 365, em seguida efetuada a Média \pm Desvio Padrão. Para a análise estatística os dados de qualidade foram realizados utilizando o programa GRAPHPAD PRISM 7,0, de 2016 XLSTAT (Addinsoft, Paris, França), os dados foram submetidos à análise de variância (ANOVA) em triplicata e as médias comparadas pelo teste de Dunnett ao nível de 5\% de significância.

\section{Resultados e Discussão}

\subsection{Caracterização das massas alimentícias frescas enriquecidas - FCA e FCB}

A caracterização físico-química e composição proximal das massas alimentícias elaboradas com farinha de caroço de açaí e farinha de casca de bacaba estão apresentadas na Tabela 3. 
Tabela 3. Caracterização físico-química e composição proximal das formulações de massa alimentícia elaborada com farinha do caroço de açaí (FCA) e farinha de casca de bacaba (FCB).

\begin{tabular}{|c|c|c|c|c|c|}
\hline Parâmetro & MC & $\begin{array}{c}\text { FCA } \\
\text { F1 } \\
\mathbf{5 \%} \\
\end{array}$ & $\begin{array}{c}\text { FCA } \\
\text { F2 } \\
10 \% \\
\end{array}$ & $\begin{array}{c}\text { FCB } \\
\text { F1 } \\
5 \% \\
\end{array}$ & $\begin{array}{c}\text { FCB } \\
\text { F2 } \\
10 \% \\
\end{array}$ \\
\hline $\mathrm{pH}$ & $6,34 \pm 0,01$ & $3,66 \pm 0,01 *$ & $4,02 \pm 0,02 *$ & $4,9 \pm 0,07 *$ & $5,05 \pm 0,14 *$ \\
\hline $\operatorname{ATT}(\%)^{* *}$ & $0,31 \pm 0,01$ & $0,52 \pm 0,02 *$ & $0,62 \pm 0,014 *$ & $0,76 \pm 0,01 *$ & $0,80 \pm 0,05^{*}$ \\
\hline SST ( ${ }^{\circ}$ Brix) & $2,3 \pm 0,28$ & $2,06 \pm 0$ & $1,93 \pm 0,14$ & $1,74 \pm 0,04$ & $3,4 \pm 0,56 *$ \\
\hline 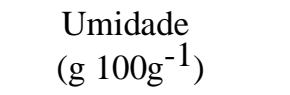 & $33,48 \pm 0,41$ & $33,80 \pm 0,07$ & $34,91 \pm 0,04 *$ & $33,76 \pm 0,07$ & $34,63 \pm 0,23 *$ \\
\hline $\begin{array}{l}\text { Lipídeos } \\
\left(\mathrm{g} 100 \mathrm{~g}^{-1}\right)\end{array}$ & $0,81 \pm 0$ & $0,46 \pm 0,00 *$ & $0,44 \pm 0,041 *$ & $2,17 \pm 0,04 *$ & $2,23 \pm 0,04 *$ \\
\hline 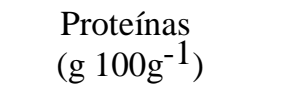 & $5,64 \pm 0,57$ & $6,23 \pm 0,11$ & $6,58 \pm 0,07 *$ & $4,95 \pm 0,39$ & $5,27 \pm 0,11$ \\
\hline 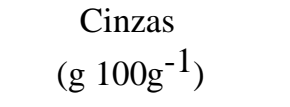 & $0,54 \pm 0$ & $0,53 \pm 0,03 *$ & $0,59 \pm 0,01 *$ & $0,58 \pm 0,00$ & $0,54 \pm 0,15$ \\
\hline $\begin{array}{l}\text { Fração Glicídica (g } \\
\left.\qquad 100 \mathrm{~g}^{-1}\right)\end{array}$ & $60,00 \pm 0,05$ & $57,26 \pm 0,45^{*}$ & $56,31 \pm 0 *$ & $57,61 \pm 0,35^{*}$ & $56,46 \pm 0,07 *$ \\
\hline 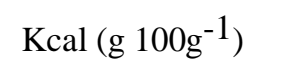 & $269,85 \pm 0,83$ & $258,73 \pm 0,21 *$ & $257,11 \pm 0,06^{*}$ & $269,77 \pm 0,62$ & $267,07 \pm 0,54$ \\
\hline
\end{tabular}

* Médias seguidas de asterisco diferem significativamente do controle pelo teste de Dunnett $(\mathrm{p}<0,05)$; **Acidez titulável total (g de ácido cítrico $100 \mathrm{~g}^{-1}$ ); *Massa controle - (MC). Fonte: Autores.

Houve uma diferença significativa das formulações com o controle para o parâmetro de pH e acidez. Em relação ao $\mathrm{pH}$, conforme as porcentagens das farinhas aumentavam, o $\mathrm{pH}$ diminuía esse fato pode ser explicado devido às farinhas FCA e FCB apresentarem valores com teor baixos de pH de 5,27 e 4,23. Sanguinetti et al. (2015) avaliaram a influência incorporação da goma xantana e goma guar no macarrão isento de glúten observaram um valor de pH variando de 5,30 a 5,60. Leite et al. (2018) observou-se em seus tratamentos que a redução do pH foi correspondente ao aumento da porcentagem da farinha de berinjela. Sendo assim, o efeito pH em massa alimentícia pode variar conforme o tipo de farinha utilizada.

Já para acidez total titulável (ATT), com adição da FCA e FCB nas formulações, ocorreu um aumento nos valores obtidos comparados ao controle. De acordo com a legislação RDC n 93/00 da ANVISA, esse parâmetro para massa alimentícia fresca ou seca pode ser no máximo $5 \mathrm{~g} / 100 \mathrm{~g}$ de acidez (Brasil, 2000).

Observa-se que houve diferença significativa $(\mathrm{p}<0,05)$ apenas entre a formulação com $10 \%$ FCB e a controle para SST, que apresentou uma concentração maior de açúcares e sais.

Já para acidez total titulável (ATT), com adição da FCA e FCB nas formulações, ocorreu um aumento nos valores obtidos comparados ao controle.

O percentual de umidade está relacionado com a sua estabilidade e composição, e pode afetar a estocagem, embalagem, processamento e principalmente a qualidade do alimento (Fujil, 2015). Em relação ao teor de umidade, à resolução RDC n 93/00 da ANVISA preconiza no máximo, para massas úmidas ou frescas, o valor de 35\% (Brasil, 2000). Portanto, os resultados obtidos para este parâmetro no presente estudo apresentaram que todas as formulações estão em acordo com a legislação brasileira. Houve uma diferença significativa $(\mathrm{p}<0,05)$ na umidade entre a massa controle e as formulações de 10\% FCA e FCB. Ribeiro et al. (2018) para formulação da massa fresca sem glúten com adição de 10\% de farinha de maracujá um valor semelhante ao encontrado neste trabalho. Oliveira et al. (2016) reportaram que a presença de fibra na farinha de 
maracujá amarelo promoveu uma maior retenção de água e, dessa forma, aumentou o teor de umidade dos produtos alimentícios.

Para o teor de lipídeos, houve diferença significativa $(\mathrm{p}<0,05)$ entra todas as formulações com substituição da farinha de arroz referente a massa controle. Segundo a Resolução no 54 de novembro de 2012 (Brasil, 2012), o teor máximo de lipídeos numa massa é de 3g/100g. Desta maneira, os valores encontrados estão de acordo com os critérios estabelecidos pela legislação vigente. Os valores superiores encontrados para as massas alimentícias com FCB que variaram de 2,17 a 2,23 (g

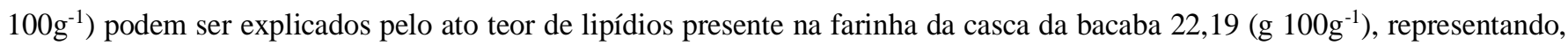
sobretudo, por composto de ácidos palmítico, oleico e linoleico, os quais estão associados a benefícios à saúde (Santos et al., 2017; Seixas et., 2016).

Os valores de cinzas apresentaram diferença estatisticamente nas formulações com FCA em relação com o controle. Observa-se que o valor de cinzas foi influenciado pela adição da FCA em que a formulação F1 apresentou diferença estatística quando comparada a F2, inversamente proporcional. Para RDC n ${ }^{\circ}$ 93/00 da ANVISA é permitido 0,65\% de cinzas nas massas frescas, logo, as massas alimentícias estão de acordo com a legislação brasileira.

Silva et al. (2015) relata que quanto menor os valores presentes nas cinzas em produtos de panificação melhor é a qualidade tecnológica do produto final. Portanto, os resultados encontrados nas formulações enriquecidas com FCA e FCB deste presente trabalho demonstraram que as quantidades encontradas variaram entre 0,53 e 0,54 indicando uma melhor consistência nas massas alimentícias. As cinzas representam minerais que compõem os alimentos, sendo eles (sódio, potássio, magnésio e cálcio, além de outros micronutrientes e macronutrientes), esses elementos são essenciais que devem ser consumidos diariamente para manutenção do organismo (Becker et al., 2018).

Quanto ao teor de proteínas, vale ressaltar que o maior valor $(\mathrm{p}<0,05)$ da formulação de $10 \%$ FCA pode estar

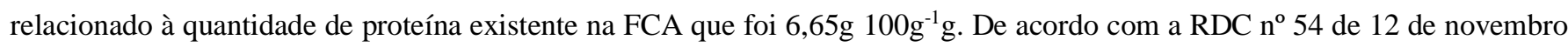
de 2012, da ANVISA (Brasil, 2012), um alimento é considerado como fonte de proteína quando apresentar no mínimo $6 \mathrm{~g}$ de proteína por porção do produto. Ambas formulações adicionadas de FCA estão dentro do padrão estabelecido pela legislação brasileira. Já as massas alimentícias enriquecidas FCB mostraram valores menores permito pela legislação brasileira. As formulações de FCB variaram porções de (5\% e 10\%), comparado com a farinha de arroz, a qual obteve maior percentual de ingrediente na massa formulada, a mesma apresenta um valor $(10,34 \pm 0,14)$ de proteína, apesar disso não foi suficiente para encontrar valor proteico na massa alimentícia (Franco et al., 2018).

$\mathrm{O}$ controle mostrou diferença significativa entre as formulações enriquecidas FCA e FCB em relação à fração glicídica. Assim, a análise dos resultados permite dizer que massa alimentícia fresca- tipo talharim, de FCA variaram entre 57,26 e 56,37 e para FCB 57,61 e 56,46 (g 100 g $\left.{ }^{-1}\right)$. De acordo com o estudo de Minguita, et al. (2015), os autores reportaram que as frações glicídicas representam as maiores quantias encontradas nas formulações, o que já era esperado, pois as massas alimentícias são satisfatória fonte de fração glicídica e pertence ao grupo de alimentos energéticos que compõe a base da pirâmide alimentar.

Em relação aos valores energéticos totais as formulações apresentaram $258,73 \mathrm{~g}\left(100 \mathrm{~g}^{-1}\right)$ e $257,11\left(\mathrm{~g}_{\left.100 \mathrm{~g}^{-1}\right)}\right)$ para farinha do caroço de açaí, apresentaram diferença significativa da massa controle. Já para as massas alimentícias adicionadas com a farinha da casca da bacaba, não apresentaram diferença significativa com a controle. Conforme a ANVISA (1998), um produto para ser considerado fonte de energia, no mínimo precisar ter $40 \mathrm{kcal}$ por $100 \mathrm{~g}$ sólido. Neste contexto, observou-se que as massas alimentícias enriquecidas com a FCA e FCB podem ser vistas como um alimento rico em calorias. 


\subsection{Análises tecnológicas das massas alimentícias}

Em relação aos resultados tecnológicos (Tabela 4), os tempos de cozimento (TOC) das massas com FCA e FCB não apresentaram diferença significativa a massa controle ( $p>0,05)$. Os valores encontrados no presente estudo foram semelhantes aos resultados obtidos para massa sem glúten preparada com o mix de farinhas elaborada por Ferreira et al. (2016) (11 a 13 min), mas foram superiores aos relatados por Ribeiro et al. (2018) que reportaram 9,53 a 10,36 min para massa fresca sem glúten com adição de farinha da casca de maracujá.

Tabela 4. Caracterização tecnológica das formulações da massa alimentícia elaborada com farinha do caroço de açaí (FCA) e com a farinha da casca da bacaba (FCB).

\begin{tabular}{cccccc}
\hline $\begin{array}{c}\text { Parâmetro } \\
\text { Químico }\end{array}$ & MC & FCA & FCA & FCB & FCB \\
& & F1 & F2 & F1 & F2 \\
\hline TOC (min) & $12,66 \pm 0$ & $12,66 \pm 0$ & $12,33 \pm 0$ & $12,66 \pm 0$ & $12,66 \pm 0$ \\
AM $(\%)$ & $90,60 \pm 0,74$ & $59,68 \pm 0,24^{*}$ & $59,40 \pm 0,31^{*}$ & $58,31 \pm 0,91^{*}$ & $76,02 \pm 0,45^{*}$ \\
\hline
\end{tabular}

* Médias seguidas de asterisco diferem significativamente do controle pelo teste de Dunnett $(\mathrm{p}<0,05)$.

* Massa controle (MC); Fonte: Autores (2021).

Quanto ao aumento da massa (AM), as massas suplementadas com FCA e FCB obtiveram uma diminuição comparada a massa controle, apresentando diferença estatística $(\mathrm{p}<0,05)$, com valores inferiores a $100 \%$. O AM é provocado pela água absorvida pelo amido durante a cocção, uma vez que ela é consumida durante o processo de gelatinizarão (Leite et al., 2018; Alonso et al., 2000). Segundo Bruneel (2010) para que uma massa alimentícia possa ter uma boa absorção o ganho da massa tem que ser duas vezes o seu peso inicial, ou seja, um ganho de $200 \%$. Desta forma, observou-se que as massas alimentícias apresentaram um menor aumento significando uma capacidade inferior de absorver água, resultando em massa firme, é o famoso ponto al dente que indica a massa cozida.

No cenário atual, o consumidor procura alimentos mais práticos, rápidos e com qualidades nutricionais. Portanto, o tempo de cozimento das massas alimentícias favorece a escolha no momento da compra do produto. Todas as formulações apresentaram o TOC em torno de 12 minutos, mostrando rapidez no preparo de uma massa isenta de glúten.

\subsection{Análise de Cor}

Na Tabela 5, estão representados os parâmetros avaliados de cor: luminosidade $\left(\mathrm{L}^{*}\right)$, coordenadas a* e $\mathrm{b}^{*}$, chroma $\left(\mathrm{C}^{*}\right)$ e ângulo Hue $\left(\mathrm{H}^{*}\right)$ tanto para as massas cruas quanto cozidas. Os resultados da ANOVA pelo teste Dunnett mostrou que algumas variáveis referentes as porcentagens das massas alimentícias com FCA e FCB diferiram significativamente $(\mathrm{p} \leq 0,05)$ da massa controle. 
Tabela 5. Análise de cor das formulações das massas alimentícias cruas e cozida elaborada com farinha do caroço de açaí (FCA) e com farinha da casca da bacaba (FCB).

\begin{tabular}{ccccccc}
\hline & & & FCA & FCA & FCB & FCB \\
& Parâmetro & MC & F1 & F2 & F1 & F2 \\
& & & $\mathbf{5 \%}$ & $\mathbf{1 0 \%}$ & $\mathbf{5 \%}$ & $\mathbf{1 0 \%}$ \\
\hline $\mathrm{L}^{*}$ & Crua & $50,13 \pm 0,08$ & $49,62 \pm 0,34$ & $41,69 \pm 0,89^{*}$ & $30,42 \pm 0,36^{*}$ & $38,97 \pm 0,95^{*}$ \\
& Cozida & $47,26 \pm 2,0$ & $22,82 \pm 1,28^{*}$ & $28,15 \pm 2,31^{*}$ & $24,95 \pm 0,21^{*}$ & $21,94 \pm 0,81^{*}$ \\
$\mathrm{a}^{*}$ & Crua & $0,74 \pm 0,02$ & $5,40 \pm 0,04^{*}$ & $8,02 \pm 0,48^{*}$ & $1,74 \pm 0,21$ & $3,09 \pm 0,02^{*}$ \\
& Cozida & $-0,37 \pm 0,31$ & $4,41 \pm 0,32^{*}$ & $5,55 \pm 0,48^{*}$ & $1,59 \pm 0,04^{*}$ & $3,46 \pm 0,11^{*}$ \\
$\mathrm{~b}^{*}$ & Crua & $8,31 \pm 0,71$ & $10,05 \pm 0,03^{*}$ & $12,60 \pm 0,85^{*}$ & $6,25 \pm 0,32^{*}$ & $7,14 \pm 0$ \\
& Cozida & $5,76 \pm 0$ & $5,14 \pm 0,54$ & $5,79 \pm 0,38$ & $4,45 \pm 0,02^{*}$ & $5,17 \pm 0,14$ \\
$\mathrm{C}^{*}$ & Crua & $9,58 \pm 0,65$ & $11,80 \pm 0,82^{*}$ & $14,06 \pm 0,39^{*}$ & $9,24 \pm 0,77$ & $7,43 \pm 0,3 *$ \\
& Cozida & $4,48 \pm 0,14$ & $7,05 \pm 0,02^{*}$ & $6,78 \pm 0,19^{*}$ & $4,9 \pm 0,37$ & $5,72 \pm 0,39$ \\
$\mathrm{H}^{*}$ & Crua & $86,12 \pm 0,07$ & $62,04 \pm 0,03^{*}$ & $58,53 \pm 0,62^{*}$ & $74,23 \pm 0,35^{*}$ & $65,38 \pm 0,13^{*}$ \\
& Cozida & $91,55 \pm 1,84$ & $50 \pm 0,68^{*}$ & $50,48 \pm 2,77^{*}$ & $69,51 \pm 0,36^{*}$ & $56,34 \pm 0,31^{*}$ \\
\hline
\end{tabular}

* Médias seguidas de asterisco diferem significativamente do controle pelo teste de Dunnett $(\mathrm{p}<0,05)$. Fonte: Autores.

Para variável $\mathrm{L}^{*}$ todas as formulações apresentadas na tabela 5 foram diferentes estatisticamente em relação a controle, tanto para massa crua quanto cozida, exceto a formulação de 5\% FCA para a massa crua. Todas apresentaram valores baixos apontando para escala escura, isso já era esperado devido a coloração dos resíduos (bacaba e açaí) por conter uma cor escura, tons roxos.

A formulação crua de 5\% FCA apresentou valores próximos ao controle para massa crua considerando o parâmetro de $\mathrm{L}^{*}$, tal similaridade pode estar relacionada a baixa adição do resíduo da farinha na massa e ao tom mais avermelhado que a farinha do caroço de açaí possui. Em massas cozidas ocorre o escurecimento da massa alimentícia devido a propriedades que geralmente afetam o escurecimento da cor como a exposição à temperatura gerando perdas de compostos que refletem na cor do produto final após cocção (Silva et al, 2019).

$\mathrm{O}$ croma $\mathrm{a}^{*}$ representa a tonalidade vermelho-verde, valores positivos significam coloração avermelhada. $\mathrm{O}$ croma $\mathrm{b}^{*}$ representa a tonalidade amarelo-azul, valores positivos para $b^{*}$ são apresentados para cor amarela e quando são elevados representa a cor dourado (Araújo et a., 2015).

Em todas as formulações, os valores foram positivos, porém baixos devido às amostras apresentarem pouca tonalidade em razão às características das matérias-primas. Portanto, encontram-se variações significativas $(p<0,05)$ entre as massas alimentícias enriquecidas de FCA e FCB com a massa controle para as massas cruas e cozidas, exceto a formulação de 5\% FCB crua. Foi possível observar a predominância da cor vermelha em todas, isso já era esperado devido à massa controle ter $100 \% \%$ da farinha de arroz que realça a cor branca. Já a massa controle cozida apresentou tom de verde após o cozimento, isso pode estar relacionado à lixiviação e degradação térmica de pigmentos (Petitot et al., 2010).

Conforme teste de Dunnett, a coordenada b* para as massas cruas de 5\% FCA e FCB e 10\% FCA mostraram que diferiram da amostra controle. Quanto à tonalidade amarelo $\left(\mathrm{b}^{*}\right)$, a massa que apresentou o maior resultado foi $10 \%$ FCA. Essas diferenças nas massas cruas podem ser explicadas devido aos diferentes tamanhos das partículas das farinhas que influenciam nas variáveis de cor (Ahmed; Al-Jassar \& Thomas, 2015). Para massa cozida, houve diferença significativa $(\mathrm{p}<0,05)$ entre a formulação de $5 \% \mathrm{FCB}$, a massa que obteve o menor valor resultando numa tonalidade menos amarelada.

De acordo com Pathare et al. (2013), a variável C* representa a intensidade da cor. Quanto maior o resultado, maior será a intensidade da cor. O ângulo Hue $\left(\mathrm{H}^{*}\right)$ representado por ângulos de $0^{\circ}$ a $360^{\circ}$. Duarte et al. (2017) relatam em seu estudo, que entre $0^{\circ}$ e $90^{\circ}$, a tonalidade que predomina são os tons em vermelhos, laranja e amarelo; de $90^{\circ}$ e $180^{\circ}$ predominam 
tons em amarelo, amarelo-esverdeado a verde; entre $180^{\circ}$ e $270^{\circ}$, predominam tons em verde, azul esverdeado e azul; entre $270^{\circ}$ e $360^{\circ}$, predominam tons em azul, púrpura, magenta e vermelho.

Neste estudo, pode-se observar, que a variação de $C^{*}$ entre 11,80 e 14,06 para as massas cruas e entre 7,05 e 6,78 para massas cozidas enriquecidas com FCA, apresentando diferença significativa $(p<0,05)$ entre a massa controle. Constata-se que as amostras enriquecidas de FCA, mostrou bons valores de saturação e a amostra com maior intensidade de cor foi a com $10 \%$ FCA, de acordo com a Fig. 1. Para as formulações cruas de FCB, apenas a com 10\% de substituição diferiu estatisticamente da formulação controle $(p<0,05)$, quanto mais próximo do valor "0" for o $C^{*}$, mais escura e menos pura e intensa é a amostra. $O$ ângulo hue $\left(\mathrm{H}^{*}\right)$ diminuiu para todas as massas alimentícias com FCA ou FCB, indicando à tonalidade vermelho puro, mostrando que houve diferença significativa com o controle $(\mathrm{p}<0,05)$.

Figura 1. Fotografia das cinco formulações (MC, 5\% FCA, 10\% FCA, 5\% FCB e 10\% FCB) das massas alimentícias frescas cruas

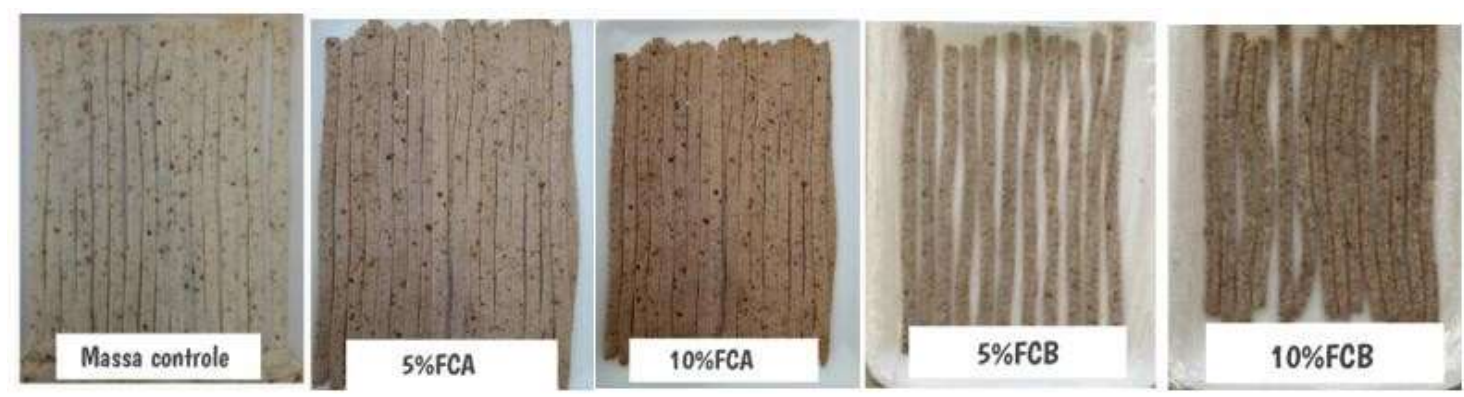

*Massa controle (MC). Fonte: Autores (2021).

\subsection{Propriedades de textura das massas alimentícias (TPA)}

A Tabela 6 apresenta os dados obtidos em relação às propriedades de textura das massas alimentícias cozidas elaboradas com FCA e FCB. Houve diferenças significativas a 5\% de probabilidade entre as amostras elaboradas FCA e FCB comparadas com a massa controle, em relação aos parâmetros força de cisalhamento, dureza, adesividade, elasticidade, gomosidade, mastigabilidade e resiliência.

Tabela 6. Propriedades de textura das formulações da massa alimentícia cozida elaborada com farinha do caroço de açaí (FCA) e com farinha da casca da bacaba (FCB).

\begin{tabular}{|c|c|c|c|c|c|}
\hline Parâmetro & $\begin{array}{c}\text { MC } \\
\text { Fo }\end{array}$ & $\begin{array}{c}\text { FCA } \\
\text { F1 } \\
\mathbf{5 \%}\end{array}$ & $\begin{array}{c}\text { FCA } \\
\text { F2 } \\
10 \%\end{array}$ & $\begin{array}{c}\text { FCB } \\
\text { F1 } \\
5 \%\end{array}$ & $\begin{array}{c}\text { FCB } \\
\text { F2 } \\
10 \%\end{array}$ \\
\hline Força Cisalhamento (gf) & $4,19 \pm 0,07$ & $4,12 \pm 0,13$ & $5,24 \pm 0,07^{*}$ & $9,91 \pm 0,15^{*}$ & $5,46 \pm 0,39^{*}$ \\
\hline Dureza $(\mathrm{N})$ & $331,89 \pm 11,2$ & $805,07 \pm 0,50 *$ & $383,73 \pm 3,35^{*}$ & $712,43 \pm 0,23 *$ & $453,522 \pm 0,77 *$ \\
\hline Adesividade (g.s) & $-18,00 \pm 0,72$ & $-14,27 \pm 0,94$ & $-34,67 \pm 0 *$ & $-34,34 \pm 1,58 *$ & $-45,04 \pm 0,46^{*}$ \\
\hline Elasticidade (mm) & $0,81 \pm 0,05$ & $0,78 \pm 0,03$ & $0,74 \pm 0,05$ & $0,78 \pm 0,12$ & $0,64 \pm 0,04 *$ \\
\hline Coesividade & $0,48 \pm 0,01$ & $0,54 \pm 0,02$ & $0,46 \pm 0,04$ & $0,57 \pm 0,02$ & $0,42 \pm 0,01$ \\
\hline Gomosidade (g) & $124,22 \pm 1,37$ & $374,58 \pm 1,59 *$ & $265,83 \pm 0,95 *$ & $472,35 \pm 1,59 *$ & $166,93 \pm 2,22 *$ \\
\hline Mastigabilidade (g) & $104,59 \pm 0,05$ & $335,88 \pm 0,41 *$ & $228,13 \pm 0,68 *$ & $394,78 \pm 1,9 *$ & $128,76 \pm 0,52 *$ \\
\hline Resiliência & $0,25 \pm 0,06$ & $0,23 \pm 0,03$ & $0,18 \pm 0,01 *$ & $0,27 \pm 0,73$ & $0,24 \pm 0,05$ \\
\hline
\end{tabular}

* Médias seguidas de asterisco diferem significativamente do controle pelo teste de Dunnett ( $<<0,05)$. *Massa controle (MC). Fonte: Autores.

A força do cisalhamento é um parâmetro que indica a força necessária para romper ou fraturar o produto. Houve diferença significativa entre a massa alimentícia com 10\% da FCA, 5\% e 10\% da FCB em relação à massa controle. Percebe-se 
que quando aumentou a concentração das farinhas ocorreu também um aumento na força de cisalhamento. Zuffo (2019) avaliou no macarrão tipo talharim com substituição parcial da farinha de trigo por farinha do mesocarpo de babaçu e encontrou valores entre 129 a 102, resultados superiores referentes ao trabalho proposto. O mesmo autor afirmar que força do cisalhamento qualifica a resistência do produto ao corte ou mordida, desse modo, quanto menor valor for encontrado, menor é a força necessária para romper o produto. Neste contexto, a massa alimentícia com 5\% da FCA, demonstrou um valor (4,12 gf) precisará de menos força para mastigar o alimento.

A dureza é um parâmetro que explica de forma mecânica, este mecanismo simula a força necessária para comprimir a amostra entre os dentes molares (para alimentos sólidos) ou entre a língua e a parte superior da boca (céu) para alimentos semi-sólidos. (SZCZESNIAK, 2002). Constata-se na tabela 6 diferenças significativas entre a formulação controle, sendo assim, a medida que houve o aumento do percentual de farinhas FCA e FCB nas massas alimentícias promoveu um aumento de dureza. Bouasla et al. (2017) descreve que está atitude pode estar relacionada com a quantidade de proteínas existente na farinha que consequentemente pode influenciar na dureza da massa. Um alimento que contém proteínas ao ser aquecido rompe-se as ligações entre as moléculas de aminoácidos modificando a sua estrutura fazendo que as proteínas ganham mais energias e, assim o alimento tendo contato com a temperatura fica com aspecto mais duro.

Gasparre; Betoret e Rosell (2019), analisaram diversas formulações de massas sem glúten comerciais e encontraram valores bastantes superiores, visto que o maior valor foi de 8366g para dureza. Neste sentido, a formulação com 10\% de FCA foi a massa que apresentou menor valor, sugerindo que esta seja a menos dura entre as amostras. No entanto, as massas alimentícias se comportam de forma diferentes de acordo com os tipos de farinhas utilizadas, vale ressalta que a quantidade de proteínas existente na farinha também influencia na qualidade da dureza com relação à massa alimentícia.

A adesividade está relacionada à pegajosidade referente a quantidade de amido solto durante o cozimento, que depois de soltar da massa e pode vir a recobri-la. Estudos afirmam que quanto maior adesividade maior a pegajosidade (Gasparre; Betoret \& Rosell, 2019). Nota-se que os valores obtidos em todas as formulações foram negativos, apresentando diferença estatística entre as formulações $10 \%$ enriquecidas de FCA e as formulações de FCB com a controle. Consequentemente, adesividade das formulações não obteve o resultado esperado, pois as massas não aderiram facilmente pegajosidade. Pesquisadores relataram que a pegajosidade é influenciada pela granulação de matérias-primas, pelo teor de fibra e pela falta de uniformidade no tamanho da partícula da farinha, causando a formulação irregular e o alimento acaba absorvendo água uniformemente (Carvalho et al, 2012).

Para o parâmetro de elasticidade as massas alimentícias não deferiram em relação a massa controle, exceto a formulação de 10\% FCB. A elasticidade está relacionada à capacidade que o alimento retorna a sua forma original depois de sofrer uma deformação. Este parâmetro está associado com a resiliência, sendo definido como uma massa resistente com a finalidade de reconstruir a forma original e, também com a qualidade do produto, mostrando quando maior o valor melhor a elasticidade (Szczesniak, 2002; Sanz et al. 2009). Percebe-se que a formulação 10\%FCB, não apresentou uma elasticidade satisfatória.

Já a coesividade é definida pela força que mantém a massa íntegra ou coesa e, também não obtiveram diferença significativa para este parâmetro, indicando que a coesividade entre as formulações adicionadas com FCA e FCB comportou-se de maneira semelhante a formulação controle.

A gomosidade é o resultado do produto entre a dureza e a coesividade. É que designa a energia necessária para fragmentar um alimento semissólido até o momento que possa engolir (Gonzalez; Alvis \& Arrazola, 2015). Referindo-se a este parâmetro as formulações enriquecidas com FCA e FCB mostraram diferença estatística significativa comparada com a controle $(\mathrm{p}<0,05)$. Se tratando da formulação com 5\%FCB, certifica-se que o seu valor foi de 472,35 (g) precisará de uma flexibilidade maior no momento de despedaçar o alimento, quanto maior o seu valor maior a dureza do produto. 
A mastigabilidade é indicada como uma energia requerida para mastigar uma amostra (velocidade constante) e reduzila a consistência até que possa engolir (Bitencourt et al., 2014). Segundo os dados obtidos pela análise estatística, o tempo de mastigação das amostras adicionada com FCA e FCB, houve diferença significativa referente ao controle ( $<<0,05)$. Sendo assim, as massas alimentícias fortalecidas tanto para FCA quanto para FCB, precisariam atribuir uma força maior no momento de mastigar o alimento. Nota-se também que a formulação com 5\% FCB teve o resultado semelhante obtido na gomosidade (394,78g), conclui-se que essa amostra precisará de mais tempo para mastigar o alimento em relação as outras.

A resiliência indica a capacidade de recuperar o estado inicial após a força de compressão. A formulação de 10\% FCA foi a única que diferiu estatisticamente $(p<0,05)$ da amostra controle. Matos, Sanz e Rosell (2014) relataram que este parâmetro pode estar relacionado aeração, frescor e a elasticidade do produto final. Neste contexto, a massa alimentícia com 10\% de FCA continha uma quantidade maior de FCA e, consequentemente mais fibra, assim resultou numa menor elasticidade, dificultando que a sua massa volte no formato original.

\section{Conclusão}

Conclui-se que o estudo das propriedades de resíduos do caroço do açaí e da casca da bacaba contribuíram para a elaboração de uma massa alimentícia fresca tipo talharim com tais características proteica, lipídios e carboidratos, exigidas pelo consumidor. A coloração das massa apresentou tonalidade vermelha, o que tornou-se um ponto positivo, pois os consumidores associam a cor mais escura com produto artesanal e mais saudável. Para o cozimento e o perfil de textura, as massas alimentícias apresentaram comportamento semelhante a uma massa integral. As massas alimentícias elaboradas com diferentes concentrações da FCA e FCB proporcionaram variações de ingredientes, além de contribuir com o meio ambiente, visto que o descarte de resíduo será reduzido.

\section{Agradecimentos}

Os autores agradecem à Universidade Federal do Tocantins e a Universidade Federal do Goiás pelo apoio no desenvolvimento da pesquisa e a CNPq pelo apoio financeiro.

\section{Referências}

Abadio Finco, F. D. B., kammerer, D. R., Carle, R., Tseng, W. H., Boser, s., \& Graeve, 1. (2012). Antioxidant activity and characterization of phenolic compounds from bacaba (Oenocarpus bacaba Mart.) fruit by HPLC-DAD-MS n. Journal of agricultural and food chemistry, 60(31), 7665-7673, $10.1021 / \mathrm{jf} 3007689$

ABIMAPI (2019). Associação Brasileira de Industria de Biscoitos, Massas Alimentícias e Paes e Bolos Industriais. http://www.abimapi.com.br/macarrao.php AACC. (2000). American Association of Cereal Chemists - AACC. Approved methods of the. (10a ed.), St. Paul.

Ahmed, J., Al-Jassar, S., \& Thomas, L. A. (2015). Comparison in rheological, thermal, and structural properties between Indian Basmati and Egyptian Giza rice flour dispersions as influenced by particle size. Food Hydrocolloids, 2015. http://www.sciencedirect.com/science/article/ pii/S0268005X15000739

Alonso, R., Aguirre, A., \& Marzo, F. (2000). Effects of extrusion and processing methods on antinutrients and in vitro digestibility of protein and starch in faba and kidney beans. Food Chemister. 10.1016/S0308-8146(99)00169-7

Andrade, L. A., Nagata, C. L. P., Assumpção, G. M. P., Gonçalves, G. A. S., \& Pereira, J. (2015). Farinha desmucilada de taro utilizada na elaboração de bolos. Científica 43(3), 203-214. 10.105361/1984-5529v43n3p203-214

ANVISA (2000). Agência Nacional de Vigilância Sanitária. Resolução RDC nº 93 de 31 de outubro de 2000. http://www.anvisa.gov.br.

ANVISA (1998). Agência Nacional de Vigilância Sanitária. Portaria nº 685, de 27 de agosto de 1998 [Aprovar o Regulamento Técnico: "Princípios Gerais para o Estabelecimento de Níveis Máximos de Contaminantes Químicos em Alimentos" e seu Anexo: "Limites máximos de tolerância para contaminantes inorgânicos

AOAC (2012). Association of Official Analytical Chemistry - AOAC. Official methods of analysis Chemists. (19e ed.), Gaithersburg, p.3000.

AOAC (2005). Association of Official Analytical Chemists International - AOAC. Official methods of analysis Chemists. (16a ed.), Washington. 
Araujo, D. R., De Lucena, E. M. P., Gomes, J. P., De Figueirêdo, R. M. F., \& Silva, E. E. (2015). Características físicas, químicas e físico-químicas dos frutos da murta. Revista Verde de Agroecologia e Desenvolvimento Sustentável,10(3),11-17.

Asmeda, R., Noorlaila, A., \& Norziah, M. H. (2016). Relações de grânulos de amido danificados e distribuição de tamanho de partícula com pasta e perfis térmicos de farinha de arroz MR263 moída. Food Chemistry, 191, 45-51.

Becker, M. M., Mandaj I, C. M., Catanante, G., Marty, J. \& L., Nunes, G. S. (2018). Mineral and bromatological assessment and determination of the antioxidant capacity and bioactive compounds in native Amazon fruits. Brazilian Jornal off Food Technology, $21,19$.

Bitencourt, C., Dutra, F. L. G., Pinto, V. Z., Helbig, E., \& Borges, L. R. (2014). Elaboração de bolos enriquecidos com semente de abóbora: avaliação química, física e sensorial. Boletim do Centro de Pesquisa de Processamento de Alimentos, 32(1).

Bouasla, A., Wojtowicz, A., \& Zidoune, M. N. (2017). Gluten-free precooked rice pasta enriched with legumes flours: Physical properties, texture, sensory attributes and microstructure. LWT - Food Science and Technology, 75(1), 569-577.

Brasil. (2012). Ministério da Saúde. Agência Nacional de Vigilância Sanitária. Resolução RDC no54 de 12 de novembro de 2012 . Regulamento técnico sobre informação nutricional complementar. Diário Oficial da União, No 219, Brasília, 13 de novembro de 2012. Seção 1, p. 122.

Brasil. (2005). Resolução RDC no 263 de 22 de setembro de 2005. Aprova o Regulamento Técnico para produtos de cereais, amidos, farinhas efarelos, constantes do anexo desta Portaria. Diário Oficial União. http://bvsms.saude.gov.br/bvs/saudelegis/anvis a/2005/rdc0263_22_09_2005.html

Brasil. (2004). Ministério da Saúde. Resolução RDC nº 216, de 15 de setembro de 2004. Regulamento Técnico de Boas Práticas para Serviços de Alimentação. Diário Oficial da União; Poder Executivo, de 16 de setembro de 2004.

Brasil. (2003). Agência Nacional de Vigilância Sanitária. Resolução RDC n 360, de 23 de dezembro de 2003 . Aprova o Regulamento Técnico sobre rotulagem nutricional de alimentos embalados. Diário Oficial [da] República Federativa do Brasil. Brasília, p.4, dez. 2003. Seção 1.

Brasil. (2000). Agência Nacional de Vigilância Sanitária. Resolução - RDC n. 93, de 31 de outubro de 2000. Regulamento técnico para fixação de identidade e qualidade de massa alimentícia. Diário oficial [da] República Federativa do Brasil, Poder Executivo, Brasília-DF, 01 novembros 2000 . Seção I.

Bruneel, C., Pareyt, B., Brijs, K., \& Delcour, J. A. (2010). O impacto da rede de proteínas nas propriedades de colagem e cozimento de produtos de massa seca. Food Chemistry, 120(2), 371-378.

Carvalho, A. V., Bassinello, P. Z., Mattietto, R. A., Carvalho, R. N., Rios, A. O., \& Seccadio, L. L. (2012). Processamento e caracterização de snack extrudado a partir de farinhas de quirera de arroz e de bandinha de feijão. Brazilian Journal of Food Technology, 15(1), 72-83.

Cecchi, L., Schuster, N., Flynn, D., Bechtel, R., Bellumori, M., Innocenti, M., \& Guinard, JX (2019). Perfil sensorial e aceitação do consumidor de massa, pão e barra de granola fortificada com bagaço de oliva seco (patê): Um subproduto da produção de azeite virgem. Journal of Food Science, 84(10), 2995-3008.

De Freitas Bonomo, L., Silva, D. N., Boasquivis, P. F., Paiva, F. A., Da Costa Guerra, J. F., Martins, T. A. F., \& Grossin, N. (2014). Açaí (Euterpe oleracea Mart.) modulates oxidative stress resistance in Caenorhabditis elegans by direct and indirect mechanisms. PloS one, 9(3), e89933.

Duarte, E. L., Carlos, L. De A., Rodrigues, C. G., Andrade, R. M., \& Oliveira, K. G. (2017) Influência da liofilização sobre os carotenoides de frutos do cerrado e comportamento higroscópico dos produtos liofilizados. Revista Perspectivas Online: Biologia \& Saúde, 23(7), 22-33.

Fujil, I. A. (2015). Determinação de umidade pelo método do aquecimento direto - Técnica gravimétrica com emprego do calor. Iuni educacional. Universidade de Cuiabá. UNIC. p. 5.

Ferreira, S. M. R., de Mello, A. P., dos Anjos, M. D. C. R., Krüger, C. C. H., Azoubel, P. M., \& de Oliveira Alves, M. A. (2016). Utilization of sorghum, rice, corn flours with potato starch for the preparation of gluten-free pasta. Food Chemistry, 191, 147-151.

Gasparre, N., Betoret, E., \& Rosell, C. M. (2019). Quality Indicators and Heat Damage of Dried and Cooked Gluten Free Spaghetti. Plant Foods for Human Nutrition, 74(4), 481-488

González, A., Alvis, A., \& Arrázola, G. (2015). Efecto del recubrimiento comestible en las propiedades de trozos de batata (Ipomoea Batatas Lam) fritos por inmersión: Parte 2: Propiedades termofísicas y de transporte. Información tecnológica, 26(1), 103-116.

Leite, N. D., Oliveira, D.S., Santos, P. A., \& Egea, M. B. (2018). Farinha de arroz e berinjela em massa alimentícia. Segurança Alimentar Nutricional, Campinas, 25(1), 65- 75. 10.20396/san.v25i1.8650927

MAPA (2014) Ministério da Agricultura Pecuária e Abastecimento - Anuário estatístico Agroenergia, Brasília. http://www.agricultura.gov.br/vegetal/culturas/cana-de-acucar

Matos, E. M., Sanz, T., \& Rosell, C. M. Establishing the function of proteins on the rheological and quality properties of rice based gluten free muffins. Food Hydrocolloids, vol. 35, 150-158. 10.1016/j.foodhyd.2013.05.007

Minguita, A. P. D. S., Carvalho, J. L. V. D., Oliveira, E. M. M., \& Galdeano, M. C. Production and characterization of pasta from biofortified foods: wheat flour, polished rice flour and whole bean flour. Ciência Rural, 45(10), 1895-1901.

Nascimento, R. A., Do Andrade, E. L., Santana, E. B., Costa, C. M. L., \& Faria, L. J. G. (2019). Bacaba poder produced in spouted bed: an alternative source of bioactive compounds and energy food product. Brazilian J Food Technol, 22, 1-15. 10.1590/1981-6723.22918

Neves, L. T. B. C., Campos, D. C. D. S., Mendes, J. K. S., Urnhani, C. O., \& Araújo, K. G. (2015). Quality of fruits manually processed of açaí (Euterpe oleracea mart.) and bacaba (Oenocarpus bacaba Mart.). Revista Brasileira de Fruticultura. 37(3), 729-738. 10.1590/0100-2945-148/14

Nogueira, A. K. M., \& Santana, A. C. (2011). Análise de sazonalidade de preços de varejo de açaí, cupuaçu e bacaba no estado do Pará. Revista de Estudos Sociais. 11 (21), 7- 22. 
Oliveira, C. F., Gurak, P. D., Cladera-Olivera, F., \& Marczak, L. D. F. (2016). Evaluation of physicochemical, technological and morphological characteristics of powdered yellow passion fruit peel. International Food Research Journal, 23(4), 1653- 1662.

Pathare, P. B., Opara, U. L., \& Al-Said, F. A. J. (2013). Colour measurement and analysis in fresh and processed foods: a review. Food and Bioprocess Technology, vol. 6, 36-60.

Petitot, M., Boyer, L., Minier, C., \& Micard, V. (2010). Fortification of pasta with split pea and faba bean flours: Pasta processing and quality evaluation. Food Research International, 43, (2), 634-641.

Phongthai, S., D'amico, S., Schoenlechner, R., Homthawornchoo, W., Rawdkuen, S. (2017). Effects of protein enrichment on the properties of rice flour based gluten- free pasta. $L W T, 80,378-385$.

Ribeiro, T. H. S., Bolanho, B. C., Montanuci, F. D., \& Ruiz, S. P. (2018). Physicochemical and sensory characterization of gluten-free fresh pasta with addition of passion fruit peel flour. Ciência Rural, 48 (12).

Sanguinetti, A. M., Del Caro, A., Scanu, A., Fadda, C.; Milella, G., Catzeddu, P., \& Piga, A. (2016). Extending the shelf life of gluten-free fresh filled pasta by modified atmosphere packaging. LWT - Food Science and Technology, 71, 96-101. https://doi.org/10.1016/j.lwt.2016.03.010.

Sanguinetti, A. M. (2015). Gluten-free fresh filled pasta: The effects of xanthan and guar gum on changes in quality parameters after pasteurisation and during storage. LWT - Food Science and Technology, 64(2), 678-684.

Santos, M. F. G., Alves, R. E., Brito, E. S., Silva, S. M., \& Silveira, M. R. S. (2017). Quality characteristis of fruits and oils of palm native to the Brazilian Amazon. Revista Brasileira De Fruticultura, 39, 305.

Sanz, T., Salvador, A., Baixauli, R., \& Fiszman, S. M. (2009). Evaluation of four types of resistant starch in muffins. II Effects in texture, colour and consumer response. European Food Research and Techonology, 229 (2), 197-204.

Seixas, F. R. F., Sesquim, E. A. R., Raasch, G. S., Cintra, D. E. (2016). Physicochemical characteristics and lipid profile of the bacaba occurring in the western Amazon. Brazilian Journal of Food Research 7(3), 105-116, 2016. https://dx.doi.org/10.3895/rebrapa.v7n3.3806

Silva, A. F. V., Laurintino, T. K. S., De Carvalho Gomes, L. D. B., Lima, R. D., \& Ribeiro, D. S. (2015). Análise de diferentes marcas de farinhas de trigo: Teor de acidez, cor e cinzas. Revista Brasileira de Agrotecnologia, 5 (1), 18-22.

Silva, I.G., Andrad E, A.P.C., Silva, L.M.R., \& Gomes, D. S. (2019). Elaboration and sensory analysis of cookies made from avocado lump flour. Brazilian Journal of Food Technology, 22.

Soler, M., Estevez, M. C., De Lourdes Moreno, M., Cebolla, A., \& Lechuga, L. M. (2016). Label-free SPR detection of gluten peptides in urine for noninvasive celiac disease follow-up. Biosensors and Bioelectronics, 79, 158-164. 10.1016/j.bios.2015.11.097

Szczesniak, A. S. (2002). Texture is a sensory property. Food Quality and preference, 13(4), 215-225.

Zuffo, A. M. (2019). A produção do Conhecimento nas Ciências Agrárias e Ambientais 3. Atena Editora, 3. 